\title{
NONCOMPACTNESS OF FOURIER CONVOLUTION OPERATORS ON BANACH FUNCTION SPACES
}

\author{
CLÁUDIO A. FERNANDES, ${ }^{1}$ ALEXEI YU. KARLOVICH, ${ }^{2}$ and YURI I. KARLOVICH ${ }^{3}$
}

\begin{abstract}
Let $X(\mathbb{R})$ be a separable Banach function space such that the Hardy-Littlewood maximal operator $M$ is bounded on $X(\mathbb{R})$ and on its associate space $X^{\prime}(\mathbb{R})$. Suppose $a$ is a Fourier multiplier on the space $X(\mathbb{R})$. We show that the Fourier convolution operator $W^{0}(a)$ with symbol $a$ is compact on the space $X(\mathbb{R})$ if and only if $a=0$. This result implies that nontrivial Fourier convolution operators on Lebesgue spaces with Muckenhoupt weights are never compact.
\end{abstract}

\section{Introduction}

The set of all Lebesgue measurable complex-valued functions on $\mathbb{R}$ is denoted by $\mathfrak{M}(\mathbb{R})$. Let $\mathfrak{M}^{+}(\mathbb{R})$ be the subset of functions in $\mathfrak{M}(\mathbb{R})$ whose values lie in $[0, \infty]$. For a measurable set $E \subset \mathbb{R}$, its Lebesgue measure and the characteristic function are denoted by $|E|$ and $\chi_{E}$, respectively. Following [1, Chap. 1, Definition 1.1], a mapping $\rho: \mathfrak{M}^{+}(\mathbb{R}) \rightarrow[0, \infty]$ is called a Banach function norm if, for all functions $f, g, f_{n}(n \in \mathbb{N})$ in $\mathfrak{M}^{+}(\mathbb{R})$, for all constants $a \geq 0$, and for all measurable subsets $E$ of $\mathbb{R}$, the following properties hold:

$$
\begin{aligned}
& \rho(f)=0 \Leftrightarrow f=0 \text { a.e., } \rho(a f)=a \rho(f), \rho(f+g) \leq \rho(f)+\rho(g), \\
& 0 \leq g \leq f \text { a.e. } \Rightarrow \rho(g) \leq \rho(f) \quad \text { (the lattice property), } \\
& 0 \leq f_{n} \uparrow f \text { a.e. } \Rightarrow \rho\left(f_{n}\right) \uparrow \rho(f) \quad \text { (the Fatou property), } \\
& |E|<\infty \Rightarrow \rho\left(\chi_{E}\right)<\infty \\
& |E|<\infty \Rightarrow \int_{E} f(x) d x \leq C_{E} \rho(f),
\end{aligned}
$$

where $C_{E} \in(0, \infty)$ may depend on $E$ and $\rho$ but is independent of $f$. When functions differing only on a set of measure zero are identified, the set $X(\mathbb{R})$ of functions $f \in \mathfrak{M}(\mathbb{R})$ for which $\rho(|f|)<\infty$ is called a Banach function space. For each $f \in X(\mathbb{R})$, the norm of $f$ is defined by

$$
\|f\|_{X(\mathbb{R})}:=\rho(|f|) .
$$

With this norm and under natural linear space operations, the set $X(\mathbb{R})$ becomes a Banach space (see [1, Chap. 1, Theorems 1.4 and 1.6]). If $\rho$ is a Banach function

2010 Mathematics Subject Classification. Primary 47G10; Secondary 46E30.

Key words and phrases. Fourier convolution operator, compactness, Banach function space, Hardy-Littlewood maximal operator, and Lebesgue space with Muckenhoupt weight. 
norm, its associate norm $\rho^{\prime}$ is defined on $\mathfrak{M}^{+}(\mathbb{R})$ by

$$
\rho^{\prime}(g):=\sup \left\{\int_{\mathbb{R}} f(x) g(x) d x: f \in \mathfrak{M}^{+}(\mathbb{R}), \rho(f) \leq 1\right\}, \quad g \in \mathfrak{M}^{+}(\mathbb{R}) .
$$

By [1, Chap. 1, Theorem 2.2], $\rho^{\prime}$ is itself a Banach function norm. The Banach function space $X^{\prime}(\mathbb{R})$ determined by the Banach function norm $\rho^{\prime}$ is called the associate space (Köthe dual) of $X(\mathbb{R})$. The associate space $X^{\prime}(\mathbb{R})$ is naturally identified with a subspace of the (Banach) dual space $[X(\mathbb{R})]^{*}$.

Let $F: L^{2}(\mathbb{R}) \rightarrow L^{2}(\mathbb{R})$ denote the Fourier transform

$$
(F f)(x):=\widehat{f}(x):=\int_{\mathbb{R}} f(t) e^{i t x} d t, \quad x \in \mathbb{R},
$$

and let $F^{-1}: L^{2}(\mathbb{R}) \rightarrow L^{2}(\mathbb{R})$ be the inverse of $F$,

$$
\left(F^{-1} g\right)(t)=\frac{1}{2 \pi} \int_{\mathbb{R}} g(x) e^{-i t x} d x, \quad t \in \mathbb{R} .
$$

It is well known that the Fourier convolution operator $W^{0}(a):=F^{-1} a F$ is bounded on the space $L^{2}(\mathbb{R})$ for every $a \in L^{\infty}(\mathbb{R})$. Let $X(\mathbb{R})$ be a separable Banach function space. Then $L^{2}(\mathbb{R}) \cap X(\mathbb{R})$ is dense in $X(\mathbb{R})$ (see Lemma 2.2 below). A function $a \in L^{\infty}(\mathbb{R})$ is called a Fourier multiplier on $X(\mathbb{R})$ if the convolution operator $W^{0}(a):=F^{-1} a F$ maps $L^{2}(\mathbb{R}) \cap X(\mathbb{R})$ into $X(\mathbb{R})$ and extends to a bounded linear operator on $X(\mathbb{R})$. The function $a$ is called the symbol of the Fourier convolution operator $W^{0}(a)$. The set $\mathcal{M}_{X(\mathbb{R})}$ of all Fourier multipliers on $X(\mathbb{R})$ is a unital normed algebra under pointwise operations and the norm

$$
\|a\|_{\mathcal{M}_{X(\mathbb{R})}}:=\left\|W^{0}(a)\right\|_{\mathcal{B}(X(\mathbb{R}))}
$$

where $\mathcal{B}(X(\mathbb{R}))$ denotes the Banach algebra of all bounded linear operators on the space $X(\mathbb{R})$.

It is well known that the multiplication operator $a I$ by a function $a \in L^{\infty}(\mathbb{R})$ is compact on the space $L^{2}(\mathbb{R})$ if and only if $a=0$ a.e. on $\mathbb{R}$ (see, e.g., [14, Corollary 2.3.2] for the case of arbitrary nonatomic measure spaces). Since $A \mapsto$ $F^{-1} A F$ for $A \in \mathcal{B}\left(L^{2}(\mathbb{R})\right)$ is a similarity transformation, we see that the Fourier convolution operator $W^{0}(a)$ cannot be compact on $L^{2}(\mathbb{R})$ unless its symbol $a$ is trivial.

The aim of this paper is to show that a nontrivial Fourier convolution operator $W^{0}(a)$ with symbol $a \in \mathcal{M}_{X(\mathbb{R})}$ is never compact on a Banach function space $X(\mathbb{R})$ if the space $X(\mathbb{R})$ satisfies a natural condition formulated in terms of the boundedness of the Hardy-Littlewood maximal operator $M$ on the space $X(\mathbb{R})$ and on its associate space $X^{\prime}(\mathbb{R})$.

Recall that the (non-centered) Hardy-Littlewood maximal function $M f$ of a function $f \in L_{\text {loc }}^{1}(\mathbb{R})$ is defined by

$$
(M f)(x):=\sup _{Q \ni x} \frac{1}{|Q|} \int_{Q}|f(y)| d y
$$

where the supremum is taken over all intervals $Q \subset \mathbb{R}$ of finite length containing $x$. The Hardy-Littlewood maximal operator $M$ defined by the rule $f \mapsto M f$ is a sublinear operator. 
Theorem 1.1 (Main result). Let $X(\mathbb{R})$ be a separable Banach function space such that the Hardy-Littlewood maximal operator $M$ is bounded on $X(\mathbb{R})$ and on its associate space $X^{\prime}(\mathbb{R})$. Suppose that $a \in \mathcal{M}_{X(\mathbb{R})}$. Then the Fourier convolution operator $W^{0}(a)$ is compact on the space $X(\mathbb{R})$ if and only if $a=0$ almost everywhere.

As a consequence of Theorem 1.1, we formulate a corollary for the case of weighted Lebesgue spaces $L^{p}(\mathbb{R}, w)$. A measurable function $w: \mathbb{R} \rightarrow[0, \infty]$ is called a weight if the preimage $w^{-1}(\{0, \infty\})$ of the set $\{0, \infty\}$ has measure zero. Let $1<p<\infty$. The weighted Lebesgue space

$$
L^{p}(\mathbb{R}, w)=\left\{f \in \mathfrak{M}(\mathbb{R}): f w \in L^{p}(\mathbb{R})\right\}
$$

is equipped with the norm

$$
\|f\|_{L^{p}(\mathbb{R}, w)}=\left(\int_{\mathbb{R}}|f(x)|^{p} w^{p}(x) d x\right)^{1 / p} .
$$

By the well-known Muckenhoupt theorem (see, e.g., [12, Theorem 2], [2, Theorem I] and [6, Chap. IV, Theorem 2.8]), the Hardy-Littlewood maximal operator $M$ is bounded on the weighted Lebesgue space $L^{p}(\mathbb{R}, w)$ if and only if the weight $w$ belongs to the Muckenhoupt class $A_{p}(\mathbb{R})$, that is, if $w \in L_{\mathrm{loc}}^{p}(\mathbb{R}), w^{-1} \in L_{\mathrm{loc}}^{p^{\prime}}(\mathbb{R})$ and

$$
\sup _{Q}\left(\frac{1}{|Q|} \int_{Q} w^{p}(x) d x\right)^{1 / p}\left(\frac{1}{|Q|} \int_{Q} w^{-p^{\prime}}(x) d x\right)^{1 / p^{\prime}}<\infty,
$$

where $1 / p+1 / p^{\prime}=1$ and the supremum is taken over all intervals $Q \subset \mathbb{R}$ of finite length $|Q|$. Since $w \in L_{\text {loc }}^{p}(\mathbb{R})$ and $w^{-1} \in L_{\text {loc }}^{p^{\prime}}(\mathbb{R})$, the weighted Lebesgue space $L^{p}(\mathbb{R}, w)$ is a separable Banach function space and $L^{p^{\prime}}\left(\mathbb{R}, w^{-1}\right)$ is its associate space (see, e.g., [11, Lemma 2.4]). It follows immediately from (1.1) that $w \in A_{p}(\mathbb{R})$ if and only if $w^{-1} \in A_{p^{\prime}}(\mathbb{R})$. Thus $w \in A_{p}(\mathbb{R})$ is also equivalent to the boundedness of the Hardy-Littlewood maximal operator $M$ on the space $L^{p^{\prime}}\left(\mathbb{R}, w^{-1}\right)$. Combining these observations with Theorem 1.1, we arrive at the following new result.

Corollary 1.2. Let $1<p<\infty$ and $w \in A_{p}(\mathbb{R})$. Suppose $a \in \mathcal{M}_{L^{p}(\mathbb{R}, w)}$. Then the Fourier convolution operator $W^{0}(a)$ is compact on the weighted Lebesgue space $L^{p}(\mathbb{R}, w)$ if and only if $a=0$ almost everywhere.

This result can be extended to the setting of weighted variable Lebesgue spaces $L^{p(\cdot)}(\mathbb{R}, w)$ because a generalization of Muckenhoupt's theorem for these spaces is available (see [3, Theorem 1.3] and [4, Theorem 1.5]).

The paper is organized as follows. In Section 2, we collect essentially known auxiliary results about the density of nice functions in separable Banach function spaces, the Stechkin type inequality for Fourier multipliers on Banach functions spaces, and the continuous embedding of the algebra $\mathcal{M}_{X(\mathbb{R})}$ into the space $L^{\infty}(\mathbb{R})$.

In Section 3, we prove that the sequence of convolution operators $\left\{W^{0}\left(\chi_{n}\right)\right\}_{n \in \mathbb{N}}$ tends strongly to the zero operator on the space $X(\mathbb{R})$ as $n \rightarrow \infty$ if their symbols $\chi_{n}$ are characteristic functions of intervals of the form $\left[t-\delta_{n}(t), t+\delta_{n}(t)\right]$ with a fixed $t \in \mathbb{R}$ and some sequence of positive numbers $\left\{\delta_{n}(t)\right\}_{n \in \mathbb{N}}$ such that $\delta_{n}(t) \rightarrow$ 
0 as $n \rightarrow \infty$. For a compact operator $W^{0}(a)$, this implies that the sequence $\left\{W^{0}\left(\chi_{n} a\right)\right\}_{n \in \mathbb{N}}$ tends to the zero operator uniformly. This fact combined with the continuous embedding of $\mathcal{M}_{X(\mathbb{R})}$ into $L^{\infty}(\mathbb{R})$ shows that the $L^{\infty}$-norm of $a$ is "locally infinitesimal" near each point $t \in \mathbb{R}$. Finally, a compactness argument shows that the $L^{\infty}$-norm of $a$ is "globally infinitesimal", whence $a=0$ a.e. on $\mathbb{R}$, which completes the proof of Theorem 1.1.

\section{Auxiliary results}

2.1. Separability, absolute continuity of the norm and density of $C_{0}^{\infty}(\mathbb{R})$ in Banach function spaces. Following [1, Chap. 1, Definition 3.1], a function $f$ in a Banach function space $X(\mathbb{R})$ is said to have absolutely continuous norm in $X(\mathbb{R})$ if $\left\|f \chi_{E_{n}}\right\|_{X(\mathbb{R})} \rightarrow 0$ for every sequence $\left\{E_{n}\right\}_{n \in \mathbb{N}}$ of measurable sets on $\mathbb{R}$ satisfying $\chi_{E_{n}} \rightarrow 0$ a.e. on $\mathbb{R}$ as $n \rightarrow \infty$. Notice that the sets $E_{n}$ are not required to have finite measure. If all functions $f \in X(\mathbb{R})$ have absolutely continuous norm in $X(\mathbb{R})$, then the space $X(\mathbb{R})$ itself is said to have absolutely continuous norm.

From [1, Chap. 1, Corollary 5.6], one can extract the following.

Lemma 2.1. A Banach function space $X(\mathbb{R})$ is separable if and only if it has absolutely continuous norm.

As usual, let $C_{0}^{\infty}(\mathbb{R})$ denote the set of all infinitely differentiable compactly supported functions on $\mathbb{R}$.

Lemma 2.2. If $X(\mathbb{R})$ is a separable Banach function space, then the sets $C_{0}^{\infty}(\mathbb{R})$ and $L^{2}(\mathbb{R}) \cap X(\mathbb{R})$ are dense in $X(\mathbb{R})$.

Proof. Since $X(\mathbb{R})$ is separable, it has absolutely continuous norm in view of Lemma 2.1. It is easy to see that the space $Y(\mathbb{R})=L^{2}(\mathbb{R}) \cap X(\mathbb{R})$ equipped with the norm

$$
\|f\|_{Y(\mathbb{R})}=\max \left\{\|f\|_{L^{2}(\mathbb{R})},\|f\|_{X(\mathbb{R})}\right\}
$$

has absolutely continuous norm, too. Then $C_{0}^{\infty}(\mathbb{R})$ is dense in $Y(\mathbb{R})$ and in $X(\mathbb{R})$ due to $[11$, Lemma $2.10(\mathrm{~b})]$. Since $C_{0}^{\infty}(\mathbb{R}) \subset Y(\mathbb{R}) \subset X(\mathbb{R})$, we conclude that $Y(\mathbb{R})$ is dense in $X(\mathbb{R})$.

2.2. Convolution operators with symbols in the algebra $\boldsymbol{V}(\mathbb{R})$. Suppose that $a: \mathbb{R} \rightarrow \mathbb{C}$ is a function of finite total variation $V(a)$ given by

$$
V(a):=\sup \sum_{k=1}^{n}\left|a\left(x_{k}\right)-a\left(x_{k-1}\right)\right|,
$$

where the supremum is taken over all partitions of $\mathbb{R}$ of the form

$$
-\infty<x_{0}<x_{1}<\cdots<x_{n}<+\infty
$$

with $n \in \mathbb{N}$. The set $V(\mathbb{R})$ of all functions of finite total variation on $\mathbb{R}$ with the norm

$$
\|a\|_{V}:=\|a\|_{L^{\infty}(\mathbb{R})}+V(a)
$$

is a unital non-separable Banach algebra. 
Theorem 2.3. Let $X(\mathbb{R})$ be a separable Banach function space such that the Hardy-Littlewood maximal operator $M$ is bounded on $X(\mathbb{R})$ and on its associate space $X^{\prime}(\mathbb{R})$. If a function $a: \mathbb{R} \rightarrow \mathbb{C}$ has a finite total variation $V(a)$, then the convolution operator $W^{0}(a)$ is bounded on the space $X(\mathbb{R})$ and

$$
\left\|W^{0}(a)\right\|_{\mathcal{B}(X(\mathbb{R}))} \leq c_{X}\|a\|_{V}
$$

where $c_{X}$ is a positive constant depending only on $X(\mathbb{R})$.

This result follows from [9, Theorem 4.3].

For Lebesgue spaces $L^{p}(\mathbb{R}), 1<p<\infty$, inequality (2.1) is usually called Stechkin's inequality, and the constant $c_{L^{p}}$ is calculated explicitly:

$$
c_{L^{p}}=\|S\|_{\mathcal{B}\left(L^{p}(\mathbb{R})\right)}= \begin{cases}\tan \left(\frac{\pi}{2 p}\right) & \text { if } 1<p \leq 2, \\ \cot \left(\frac{\pi}{2 p}\right) & \text { if } 2 \leq p<\infty,\end{cases}
$$

where $S$ is the Cauchy singular integral operator given by

$$
(S f)(x):=\frac{1}{\pi i} \lim _{\varepsilon \rightarrow 0} \int_{\mathbb{R} \backslash(x-\varepsilon, x+\varepsilon)} \frac{f(t)}{t-x} d t .
$$

We refer to [5, Theorem 2.11] for the proof of (2.1) in the case of Lebesgue spaces $L^{p}(\mathbb{R})$ with $c_{L^{p}}=\|S\|_{\mathcal{B}\left(L^{p}(\mathbb{R})\right)}$ and to [7, Chap. 13, Theorem 1.3] for the calculation of the norm of $S$ given in the second equality in (2.2).

2.3. Continuous embedding of the algebra of multipliers $\mathcal{M}_{\boldsymbol{X}(\mathbb{R})}$ into $\boldsymbol{L}^{\infty}(\mathbb{R})$. One of the main ingredients of the proof of Theorem 1.1 is the following.

Theorem 2.4. Let $X(\mathbb{R})$ be a separable Banach function space such that the Hardy-Littlewood maximal operator $M$ is bounded on $X(\mathbb{R})$ and on its associate space $X^{\prime}(\mathbb{R})$. If $a \in \mathcal{M}_{X(\mathbb{R})}$, then

$$
\|a\|_{L^{\infty}(\mathbb{R})} \leq\|a\|_{\mathcal{M}_{X(\mathbb{R})}} .
$$

Proof. By [11, Theorem 3.8], the Cauchy singular integral operator $S$ given by (2.3) is bounded on the space $X(\mathbb{R})$. Then, in view of [11, Theorem 3.9],

$$
\sup _{-\infty<a<b<\infty} \frac{1}{b-a}\left\|\chi_{(a, b)}\right\|_{X(\mathbb{R})}\left\|\chi_{(a, b)}\right\|_{X^{\prime}(\mathbb{R})}<\infty .
$$

If (2.5) is fulfilled, then inequality (2.4) follows from [10, inequality (1.2) and Corollary 4.2].

\section{Proof of the main result}

3.1. Strong convergence to zero of convolution operators whose symbols are characteristic functions of segments shrinking to a point. For every $t \in \mathbb{R}$ and every $\delta>0$, let $\chi_{t, \delta}$ be the characteristic function of the segment $[t-\delta, t+\delta]$ and let $\chi_{t, \delta}^{*}$ be the characteristic function of $\mathbb{R} \backslash[t-\delta, t+\delta]$. We will denote by $\operatorname{s-lim}_{n \rightarrow \infty} A_{n}$ the strong limit of a sequence of operators $\left\{A_{n}\right\}_{n \in \mathbb{N}} \subset$ $\mathcal{B}(X(\mathbb{R}))$. 
Theorem 3.1. Let $X(\mathbb{R})$ be a separable Banach function space. If the HardyLittlewood maximal operator $M$ is bounded on the space $X(\mathbb{R})$ and on its associate space $X^{\prime}(\mathbb{R})$, then for every point $t \in \mathbb{R}$ and every sequence $\left\{\delta_{n}(t)\right\}_{n \in \mathbb{N}}$ of positive numbers such that

$$
\lim _{n \rightarrow \infty} \delta_{n}(t)=0
$$

we have

$$
\operatorname{s}_{n \rightarrow \infty} W^{0}\left(\chi_{t, \delta_{n}(t)}\right)=0
$$

on the space $X(\mathbb{R})$.

Proof. Fix a point $t \in \mathbb{R}$ and a sequence $\left\{\delta_{n}(t)\right\}_{n \in \mathbb{N}}$ of positive numbers satisfying (3.1). Since $\chi_{t, \delta_{n}(t)} \in V(\mathbb{R})$ for every $n \in \mathbb{N}$, it follows from Theorem 2.3 that the operator $W^{0}\left(\chi_{t, \delta_{n}(t)}\right)$ is bounded on the space $X(\mathbb{R})$ for every $n \in \mathbb{N}$. By Lemma 2.2, the set $C_{0}^{\infty}(\mathbb{R})$ is dense in $X(\mathbb{R})$. In view of this observation and a well-known fact from Functional Analysis (see, e.g., [13, Lemma 1.4.1(ii)]), in order to prove equality (3.2), it is sufficient to show that for every $f \in C_{0}^{\infty}(\mathbb{R})$,

$$
\lim _{n \rightarrow \infty}\left\|W^{0}\left(\chi_{t, \delta_{n}(t)}\right) f\right\|_{X(\mathbb{R})}=0 .
$$

Let $S(\mathbb{R})$ denote the Schwartz space of rapidly decreasing functions. If $f \in$ $C_{0}^{\infty}(\mathbb{R}) \subset S(\mathbb{R})$, then its Fourier transform $\psi:=F f$ belongs to $S(\mathbb{R})$ (see, e.g., [8, Corollary 2.2.15]). Hence

$$
K_{0}:=\max _{x \in \mathbb{R}}|\psi(x)|<\infty, \quad K_{1}:=\max _{x \in \mathbb{R}}\left|\psi^{\prime}(x)\right|<\infty .
$$

If $x \neq 0$, then integrating by parts, we get for all $n \in \mathbb{N}$,

$$
\begin{aligned}
2 \pi\left(W^{0}\left(\chi_{t, \delta_{n}(t)}\right) f\right)(x) & =\int_{t-\delta_{n}(t)}^{t+\delta_{n}(t)} e^{-i x \tau} \psi(\tau) d \tau \\
& =\left.\frac{e^{-i x \tau} \psi(\tau)}{-i x}\right|_{\tau=t-\delta_{n}(t)} ^{\tau=t+\delta_{n}(t)}+\frac{1}{i x} \int_{t-\delta_{n}(t)}^{t+\delta_{n}(t)} e^{-i x \tau} \psi^{\prime}(\tau) d \tau .
\end{aligned}
$$

It follows from (3.4) and (3.5) that for $x \in \mathbb{R}$ and $m, n \in \mathbb{N}$,

$$
\begin{aligned}
2 \pi & \left|\left(W^{0}\left(\chi_{t, \delta_{n}(t)}\right) f\right)(x)\right| \\
= & \left|\int_{t-\delta_{n}(t)}^{t+\delta_{n}(t)} e^{-i x \tau} \psi(\tau) d \tau\right| \chi_{0, m}(x)+\left|\int_{t-\delta_{n}(t)}^{t+\delta_{n}(t)} e^{-i x \tau} \psi(\tau) d \tau\right| \chi_{0, m}^{*}(x) \\
\leq & \left(\int_{t-\delta_{n}(t)}^{t+\delta_{n}(t)}|\psi(\tau)| d \tau\right) \chi_{0, m}(x) \\
& +\left(\frac{\left|\psi\left(t+\delta_{n}(t)\right)\right|+\left|\psi\left(t-\delta_{n}(t)\right)\right|}{|x|}+\frac{1}{|x|} \int_{t-\delta_{n}(t)}^{t+\delta_{n}(t)}\left|\psi^{\prime}(\tau)\right| d \tau\right) \chi_{0, m}^{*}(x) \\
\leq & 2 K_{0} \delta_{n}(t) \chi_{0, m}(x)+\left(\frac{2 K_{0}}{|x|}+\frac{2 K_{1} \delta_{n}(t)}{|x|}\right) \chi_{0, m}^{*}(x) .
\end{aligned}
$$


By [8, Example 2.1.4], if $|x|>1$, then

$$
\left(M \chi_{0,1}\right)(x)=\frac{2}{|x|+1} \geq \frac{1}{|x|} .
$$

It follows from (3.6)-(3.7) and the lattice property of the norm of $X(\mathbb{R})$ that for all $m, n \in \mathbb{N}$,

$$
\begin{aligned}
\left\|\left(W^{0}\left(\chi_{t, \delta_{n}(t)}\right) f\right)(x)\right\|_{X(\mathbb{R})} \leq & \frac{K_{0} \delta_{n}(t)}{\pi}\left\|\chi_{0, m}\right\|_{X(\mathbb{R})} \\
& +\frac{K_{0}+K_{1} \delta_{n}(t)}{\pi}\left\|\chi_{0, m}^{*}\left(M \chi_{0,1}\right)\right\|_{X(\mathbb{R})} .
\end{aligned}
$$

Since the Hardy-Littlewood maximal operator $M$ is bounded on $X(\mathbb{R})$ and since $\chi_{0,1} \in X(\mathbb{R})$, we see that $M \chi_{0,1} \in X(\mathbb{R})$. By Lemma 2.1, the function $M \chi_{0,1}$ has absolutely continuous norm in $X(\mathbb{R})$.

Fix $\varepsilon>0$. It is clear that $\chi_{0, m}^{*} \rightarrow 0$ a.e. on $\mathbb{R}$ as $m \rightarrow \infty$. It follows from the absolute continuity of the norm of $M \chi_{0,1} \in X(\mathbb{R})$ that there exists an $m \in \mathbb{N}$ such that

$$
\frac{K_{0}+1}{\pi}\left\|\chi_{0, m}^{*}\left(M \chi_{0,1}\right)\right\|_{X(\mathbb{R})}<\frac{\varepsilon}{2}
$$

On the other hand, it follows from (3.1) that there exists an $N \in \mathbb{N}$ such that for all $n>N$,

$$
K_{1} \delta_{n}(t)<1
$$

and

$$
\frac{K_{0} \delta_{n}(t)}{\pi}\left\|\chi_{0, m}\right\|_{X(\mathbb{R})}<\frac{\varepsilon}{2}
$$

Combining (3.8)-(3.11), we see that

$$
\left\|\left(W^{0}\left(\chi_{t, \delta_{n}(t)}\right) f\right)(x)\right\|_{X(\mathbb{R})}<\varepsilon \text { for all } n>N,
$$

which implies (3.3) for all $f \in C_{0}^{\infty}(\mathbb{R})$, and this completes the proof of (3.2).

3.2. Proof of Theorem 1.1. Suppose $a \in \mathcal{M}_{X(\mathbb{R})}$. If $a=0$, then $W^{0}(a)$ is the zero operator, whence it is compact.

Now assume that $W^{0}(a)$ is compact on the space $X(\mathbb{R})$. Consider an arbitrary segment $\left[x_{0}, y_{0}\right] \subset \mathbb{R}$. By Theorem 3.1, for every point $t \in\left[x_{0}, y_{0}\right]$ and every sequence of positive numbers $\left\{\delta_{n}(t)\right\}_{n \in \mathbb{N}}$ such that $\delta_{n}(t) \rightarrow 0$ as $n \rightarrow \infty$, we get equality (3.2) on the space $X(\mathbb{R})$. Since the operator $W^{0}(a)$ is compact, it follows from (3.2) and [13, Lemma 1.4.7] that for every $t \in\left[x_{0}, y_{0}\right]$,

$$
\lim _{n \rightarrow \infty}\left\|W^{0}\left(\chi_{t, \delta_{n}(t)} a\right)\right\|_{\mathcal{B}(X(\mathbb{R}))}=\lim _{n \rightarrow \infty}\left\|W^{0}\left(\chi_{t, \delta_{n}(t)}\right) W^{0}(a)\right\|_{\mathcal{B}(X(\mathbb{R}))}=0 .
$$

Fix an arbitrary $\varepsilon>0$. Then for every $t \in\left[x_{0}, y_{0}\right]$ there exists an $n(t) \in \mathbb{N}$ such that

$$
\left\|W^{0}\left(\chi_{t, \delta_{n(t)}(t)} a\right)\right\|_{\mathcal{B}(X(\mathbb{R}))}<\varepsilon
$$

Since $\left[x_{0}, y_{0}\right]$ is a compact set, we can extract a finite subcovering $\gamma_{1}, \ldots, \gamma_{m}$ from the open covering

$$
\bigcup_{t \in\left[x_{0}, y_{0}\right]}\left(t-\delta_{n(t)}(t), t+\delta_{n(t)}(t)\right)
$$


of $\left[x_{0}, y_{0}\right]$, where each interval $\gamma_{j}$ is of the form

$$
\gamma_{j}=\left(t_{j}-\delta_{n\left(t_{j}\right)}\left(t_{j}\right), t_{j}+\delta_{n\left(t_{j}\right)}\left(t_{j}\right)\right)
$$

and $t_{j} \in\left[x_{0}, y_{0}\right]$ for all $j \in\{1, \ldots, m\}$. Thus

$$
\left\|W^{0}\left(\chi_{\gamma_{j}} a\right)\right\|_{\mathcal{B}(X(\mathbb{R}))}<\varepsilon \quad \text { for all } j \in\{1, \ldots, m\} .
$$

By Theorem 2.4, for all $j \in\{1, \ldots, m\}$,

$$
\left\|\chi_{\gamma_{j}} a\right\|_{L^{\infty}(\mathbb{R})} \leq\left\|\chi_{\gamma_{j}} a\right\|_{\mathcal{M}_{X(\mathbb{R})}}=\left\|W^{0}\left(\chi_{\gamma_{j}} a\right)\right\|_{\mathcal{B}(X(\mathbb{R}))} .
$$

Since $\left[x_{0}, y_{0}\right] \subset \gamma_{1} \cup \cdots \cup \gamma_{m}$, taking into account (3.12) and (3.13), we get

$$
\left\|\chi_{\left[x_{0}, y_{0}\right]} a\right\|_{L^{\infty}(\mathbb{R})} \leq\left\|\chi_{\gamma_{1} \cup \cdots \cup \gamma_{m}} a\right\|_{L^{\infty}(\mathbb{R})}=\max _{1 \leq j \leq m}\left\|\chi_{\gamma_{j}} a\right\|_{L^{\infty}(\mathbb{R})}<\varepsilon .
$$

Passing to the limit as $\varepsilon \rightarrow 0$ in the above inequality, we see that $a(t)=0$ for almost all $t \in\left[x_{0}, y_{0}\right]$. Since $\left[x_{0}, y_{0}\right] \subset \mathbb{R}$ was chosen arbitrarily, we conclude that $a=0$ almost everywhere on $\mathbb{R}$.

Acknowledgments. This work was partially supported by the Fundação para a Ciência e a Tecnologia (Portuguese Foundation for Science and Technology) through the project UID/MAT/00297/2013 (Centro de Matemática e Aplicações).

\section{REFERENCES}

[1] C. Bennett and R. Sharpley, Interpolation of Operators, Academic Press, Boston, 1988.

[2] R. R. Coifman and C. Fefferman, Weighted norm inequalities for maximal functions and singular integrals, Studia Math. 51 (1974), 241-249.

[3] D. Cruz-Uribe, L. Diening, and P. Hästö, The maximal operator on weighted variable Lebesgue spaces, Frac. Calc. Appl. Anal. 14 (2011), 361-374.

[4] D. Cruz-Uribe, A. Fiorenza, and C. J. Neugebauer, Weighted norm inequalities for the maximal operator on variable Lebesgue spaces, J. Math. Anal. Appl. 394 (2012), 744-760.

[5] R. Duduchava, Integral Equations with Fixed Singularities, Teubner Verlagsgesellschaft, Leipzig, 1979.

[6] J. García-Cuerva and J. Rubio de Francia, Weighted Norm Inequalities and Related Topics, North-Holland, Amsterdam, 1985.

[7] I. Gohberg and N. Krupnik, One-Dimensional Linear Singular Integral Equations. Vol. II: General Theory and Applications, Birkhäuser, Basel, 1992.

[8] L. Grafakos, Classical Fourier Analysis, 3rd ed., Springer, New York, 2014.

[9] A. Yu. Karlovich, Maximally modulated singular integral operators and their applications to pseudodifferential operators on Banach function spaces, Contemp. Math. 645 (2015), $165-178$.

[10] A. Karlovich and E. Shargorodsky, When does the norm of a Fourier multiplier dominate its $L^{\infty}$ norm? Proc. London Math. Soc. 118 (2019), 901-941.

[11] A. Yu. Karlovich and I. M. Spitkovsky, The Cauchy singular integral operator on weighted variable Lebesgue spaces, Oper. Theor. Adv. Appl. 236 (2014), 275-291.

[12] B. Muckenhoupt, Weighted norm inequalities for the Hardy maximal function, Trans. Amer. Math. Soc. 165 (1972), 207-226.

[13] S. Roch, P. A. Santos, and B. Silbermann, Non-Commutative Gelfand Theories. A Tool-kit for Operator Theorists and Numerical Analysts, Springer, Berlin, 2011.

[14] R. K. Singh and J. S. Manhas, Composition Operators on Function Spaces, North-Holland, Amsterdam, 1993. 
${ }^{1}$ Centro de Matemática e AplicaÇões, Departamento de Matemática, Faculdade de Ciências e Tecnologia, Universidade Nova de Lisboa, Quinta da Torre, 2829-516 Caparica, Portugal.

E-mail address: caf@fct.unl.pt

2 Centro de Matemática e Aplicações, Departamento de Matemática, Faculdade de Ciências e Tecnologia, Universidade Nova de Lisboa, Quinta da Torre, 2829-516 Caparica, Portugal.

E-mail address: oyk@fct.unl.pt

${ }^{3}$ Centro de Investigación en Ciencias, instituto de Investigación en Ciencias Básicas y Aplicadas, Universidad Autónoma del Estado de Morelos, Av. UniverSidad 1001, Col. Chamilpa, C.P. 62209 Cuernavaca, Morelos, México.

E-mail address: karlovich@uaem.mx 